\title{
Das Management von Forschungsdaten als Handlungsfeld wissenschaftlicher Bibliotheken: Forschungsunterstützung am Beispiel ZB MED - Informationszentrum Lebenswissenschaften
}

https://doi.org/10.1515/bfp-2019-2006

Zusammenfassung: Der Beitrag ordnet das Handlungsfeld Forschungsdatenmanagement zunächst in den Kontext sich wandelnder Anforderungen an wissenschaftliche Bibliotheken sowie wissenschaftspolitischer Strategien und Forderungen auf nationaler und europäischer Ebene ein. Im Anschluss liegt der Schwerpunkt auf einem möglichen Leistungsportfolio einer wissenschaftlichen Bibliothek, das Forschenden Unterstützung bei dem Management und der Publikation ihrer Forschungsdaten bietet. Die denkbaren Serviceleistungen reichen dabei von der Beratung bis hin zum Aufbau eigener Forschungsdateninfrastrukturen, die den Nachweis, die Bewertung, das Sichtbarmachen und die Überprüfung von Forschungsdaten ermöglichen.

Schlüsselwörter: Forschungsdaten; Forschungsdatenmanagement; Infrastruktur

\section{Research Data Management as a Field of Action of Re- search Libraries - Using the Example of ZB MED - Infor- mation Centre for Life Sciences}

Abstract: The field of action "research data management" is placed within the context of changing requirements to research libraries as well as of strategies and requirements named by science policy on national and European level. The focus lies on a potential service portfolio of a research library, which offers researchers support with regard to the management and publication of research data. Potential services might range from advisory services to the development of research data infrastructures, which facilitate the record, evaluation, visibility, and review of research data.

Keywords: Research data; research data management; infrastructure

\section{Anforderungen an wissenschaftliche Bibliotheken}

Im Zuge der Digitalisierung der Wissenschaft stellen sich neben der Informations- und Literaturversorgung für wissenschaftliche Bibliotheken neue Anforderungen.

„Perspektivisch entwickeln sich Bibliotheken zu virtuellen Arbeitsumgebungen, die wissenschaftliches Arbeiten in Forschung, Lehre und Studium fachspezifisch mit attraktiven Infrastrukturdienstleistungen und Werkzeugen unterstützen und für Fachcommunities die Voraussetzungen für Interaktion und Kollaboration schaffen." ${ }^{1}$

Wichtige Aspekte hierbei sind die Begleitung und Unterstützung des gesamten Forschungsprozesses sowie die enge Anbindung an die Bedarfe der jeweiligen Fachcommunity. Ein strategisches Umdenken von „Besitz“ von Literatur hin zu „Zugang schaffen“ zu Literatur erfordert ein kooperatives Handeln der Bibliotheken untereinander. Zur Schaffung ,attraktiver Infrastrukturdienstleistungen und Werkzeuge“ ist darüber hinaus die Zusammenarbeit mit Partnern wie Rechenzentren oder Forschungsinstitutionen aus dem IT-Bereich notwendig. Einen Vorteil von Bibliotheken stellen in diesem Zusammenhang die langfristigen und nachhaltigen Finanzierungsstrukturen dar, die dazu genutzt werden sollten, projektbezogene Entwicklungen im Rahmen von Forschungsprojekten $\mathrm{zu}$ nachhaltigen Strukturen auszubauen.

Services im Rahmen des Managements von digitalen Forschungsdaten sind hier ein wichtiges Handlungsfeld im Zusammenspiel mit der Förderung digitaler Informationskompetenz.

1 Deutscher Bibliotheksverband (2018) 1.
*Kontaktperson: Birte Lindstädt, lindstaedt@zbmed.de Jasmin Schmitz, schmitz@zbmed.de 


\section{Wissenschaftspolitischer Rahmen für das Handlungsfeld Forschungsdatenmanagement}

\subsection{Nationale Ebene: Nationale Forschungsdateninfrastruktur NFDI}

Im Jahre 2014 nahm der von der Gemeinsamen Wissenschaftskonferenz eingesetzte Rat für Informationsinfrastrukturen RfII seine Arbeit auf. Ein wegweisendes Ergebnis des RfII war das 2016 veröffentlichte Papier „Leistung aus Vielfalt",'2 das das Forschungsdatenmanagement zu einem Schlüsselthema auf der Digitalen Agenda in der Wissenschaftslandschaft macht und den Aufbau einer Nationalen Forschungsdateninfrastruktur NFDI vorschlägt.

Die Ausgestaltung der NFDI wird in den Papieren „Schritt für Schritt - oder: Was bringt wer mit?“3 von 2017 und „Zusammenarbeit als Chance“ von 2018 erläutert. ${ }^{4}$ Demnach soll die NFDI im Zuge eines moderierten Prozesses entstehen, indem Infrastrukturpartner mit Partnern aus einer Fachcommunity zusammenarbeiten und entlang fachlich-thematischer Domänen Konsortien bilden.

Inhaltliche Anforderungen des RfII an die Konsortialpartner sind folgende (eigene Zusammenstellung auf Basis des Papiers des Rats für Informationsinfrastrukturen) $)^{5}$ :

Tab. 1: Inhaltliche Anforderungen des RfII

\begin{tabular}{lll}
\hline $\begin{array}{l}\text { wissenschaftliche } \\
\text { Communities }\end{array}$ & $\begin{array}{l}\text { Infrastruktureinrich- } \\
\text { tungen }\end{array}$ & gemeinsam \\
\hline $\begin{array}{l}\text { Kritische Masse } \\
\text { erreichen }\end{array}$ & Erfahrung & $\begin{array}{l}\text { Fachübergreifend, } \\
\text { interoperabel }\end{array}$ \\
\hline $\begin{array}{l}\text { Dynamik von } \\
\text { Datenproduzen- } \\
\text { ten und -nutzern } \\
\text { berücksichtigen }\end{array}$ & Serviceorientierung & $\begin{array}{l}\text { Hochschulen und } \\
\text { außeruniversitäre } \\
\text { Forschungseinrich- } \\
\text { tungen }\end{array}$ \\
\hline $\begin{array}{l}\text { Überinstitutionell, } \\
\text { überregional }\end{array}$ & $\begin{array}{l}\text { Nachhaltigkeit } \\
\text { grundlegender }\end{array}$ & $\begin{array}{l}\text { Gemeinsame Gover- } \\
\text { nance }\end{array}$ \\
\hline Selbstorganisation & Entwicklung von & $\begin{array}{l}\text { Internationale Ein- } \\
\text { und Anbindung }\end{array}$ \\
\hline $\begin{array}{l}\text { Bedarfe breit } \\
\text { ermitteln }\end{array}$ & $\begin{array}{l}\text { Expertise hinsichtlich } \\
\text { der fachlichen }\end{array}$ & $\begin{array}{l}\text { Zusammenwirken in } \\
\text { der Ausbildung von }\end{array}$ \\
& Bedarfe entwickeln & \begin{tabular}{l} 
Fachpersonal \\
\hline
\end{tabular}
\end{tabular}

2 Rat für Informationsinfrastrukturen (2016a).

3 Rat für Informationsinfrastrukturen (2016b).

4 Rat für Informationsinfrastrukturen (2018).

5 Rat für Informationsinfrastrukturen (2016b).
Tab. 1: (fortgesetzt)

\begin{tabular}{ll}
\hline $\begin{array}{l}\text { wissenschaftliche } \\
\text { Communities }\end{array}$ & $\begin{array}{l}\text { Infrastruktureinrich- } \\
\text { tungen }\end{array}$ \\
\hline $\begin{array}{l}\text { Nachnutzung } \\
\text { von Daten } \\
\text { ermöglichen }\end{array}$ & $\begin{array}{l}\text { Kollaboration und } \\
\text { Synergien schaffen }\end{array}$ \\
\hline $\begin{array}{l}\text { Auswertungs- } \\
\text { möglichkeiten } \\
\text { von Forschungs- } \\
\text { daten weiterent- } \\
\text { wickeln }\end{array}$ & \\
\hline $\begin{array}{l}\text { Qualitätsstandards } \\
\text { entwickeln und } \\
\text { einhalten }\end{array}$ & Interaktion mit \\
\hline
\end{tabular}

ZB MED hat diesen Rahmen aufgegriffen und proaktiv ein Konsortium NFDI4Life gegründet, das die Lebenswissenschaften in einem breiten Ansatz abdeckt. Ca. 20 Forschungs- und Infrastruktureinrichtungen bereiten hier eine Nationale Forschungsdateninfrastruktur für die Lebenswissenschaften vor, indem zunächst relevante Themen wie etwa Qualitätssicherung und Interoperabilität durch einheitliche Standards, Reputationsmechanismen für Aufgaben des Forschungsdatenmanagements oder Umgang mit datenschutzrelevanten Forschungsdaten im Rahmen von Anwendungsfällen aus der Forschungspraxis („use cases") aufgearbeitet werden.

\subsection{Europäische Ebene: European Open Science Cloud}

Die Rahmensetzung für eine NFDI betont die internationale Anbindungsfähigkeit einer solchen Struktur. Auf europäischer Ebene betrifft dies die Planungen für die European Open Science Cloud (EOSC), die ähnliche Forderungen aufstellt wie der RfII für die NFDI. Die Europäische Kommission stellt u. a. folgende Forderungen für die $\operatorname{EOSC}^{6}$ auf:

- Open Access by default

- FAIR-Prinzipien für Forschungsdaten beachten

- Data Stewardship

- Entwicklung von Reputationsmechanismen

- Entwicklung von Qualitätsstandards

- Einbindung bestehender Informationsinfrastrukturen

- Beachtung der Nutzerbedarfe

Ein besonderer Schwerpunkt liegt bei der European Open Science Cloud auf der Veröffentlichung von Forschungs-

6 European Commission and Union (2017). 
daten gemäß den FAIR-Prinzipien. FAIR steht dabei für findable, accessible, interoperable, re-usable. Diese Attribute sollen in erster Linie über eine umfangreiche Beschreibung der Daten mittels Metadaten sowie über die Vergabe von persistenten Identifikatoren und Lizenzen (z. B. Creative Commons) erzielt werden. Was FAIR im Einzelnen genau bedeutet und wie die Forderungen umgesetzt werden können, ist im FAIR Data Action Plan dargelegt, dessen Entwurf im Juni 2018 veröffentlicht wurde. ${ }^{7}$ Der Plan ist das Ergebnis der Arbeit einer Expertengruppe, die die Europäische Kommission im Hinblick auf die Umsetzung der FAIRPrinzipien berät. Nach einer Konsultationsphase wurde der Plan im November 2018 als Handlungsrahmen verabschiedet.

\section{Handlungsfeld} Forschungsdatenmanagement bei ZB MED - Informationszentrum Lebenswissenschaften

\subsection{Forschungsdaten in den Lebenswissenschaften}

In jeder Disziplin muss fachspezifisch festgelegt werden, was die relevanten digitalen Forschungsdaten sind. Die hier als Beispiel herangezogenen Lebenswissenschaften umfassen die Disziplinen Medizin, Biologie, Ernährungs-, Agrar- und Umweltwissenschaften. In den Agrarwissenschaften sind Forschungsdaten u. a.

- Messdaten (z. B. Sediment-Analyse-Daten, Temperatur-Angaben)

- Geodaten (z. B. GIS-Dateien)

- Genomdaten (z.B. Länge extrahierter Genome von Pflanzen)

- Interviews (z. B. mit Experten) oder

- Bilder (z. B. Fotos, Satellitenaufnahmen)

In jeder Disziplin gibt es darüber hinaus besondere Rahmenbedingungen, die im Forschungsdatenmanagement berücksichtigt werden müssen. In den Agrarwissenschaften wird die Diskussion aktuell dahingehend geführt, wie mit sog. Big Data umgegangen wird. Hier geht es um betriebsbezogene Maschinendaten, die im Zuge der Digitalisierung der Landwirtschaft von Maschinen während des Arbeitsprozesses erhoben und weitergeleitet werden. Dies

7 Hodson et al. (2018). sind zunächst einmal Rohdaten, die zunächst kommerziell von den Maschinenherstellern genutzt werden. Sie werden dann zu Forschungsdaten im o.g. Sinne, wenn die Wissenschaft Zugang zu ihnen bekommt und sie zu Forschungszwecken verwendet. Vorteile von Forschungsergebnissen aus der Auswertung von Big Data bzw. Forschungsdaten für die Landwirtschaft können z.B. eine Schonung des Feldbodens durch eine bessere Koordination der Landmaschinen sein oder - allgemein - die Lösung praktischer Probleme, um einen Beitrag zur Verbesserung der weltweiten Ernährungssituation zu leisten.

In der Medizin handelt es sich bei Forschungsdaten beispielsweise um:

- Bilddaten aus bildgebenden Verfahren (z. B. MRT)

- Sensordaten aus Biosignal- oder Vitalparametermessung (z. B. EKG, EEG)

- Biomaterialdaten aus Laboruntersuchungen (z.B. Blutproben, Genom-Daten)

- Befunddaten aus der ärztlichen Diagnostik (z.B. Anamnese)

- Statistikdaten (z.B. aus anonymisierten Befunddaten)

- Klassifikationen und Codes zu Krankheiten oder Materialien (z. B. International Statistical Classification of Diseases and Related Health Problems (ICD))

- Stammdaten der Patientenverwaltung (z. B. aus Krankenhausinformationssystemen)

Besondere Rahmenbedingungen für das Forschungsdatenmanagement in der Medizin sind u.a. die enormen Datenmengen bzw. Dateigrößen, die für eine Archivierung bzw. Publikation anfallen, was vor allem auf die Vielzahl der bildgebenden Verfahren oder auf Daten aus Gen-Sequenzierungen zurückzuführen ist. Eine weitere Besonderheit bilden die rechtlichen Rahmenbedingungen in der Medizin im Hinblick auf Datenschutz und Persönlichkeitsrechte von Patienten. ${ }^{8}$

\subsection{Leistungsspektrum Forschungsdatenmanagement bei ZB MED}

Vor dem Hintergrund verschiedener Zielsetzungen wissenschaftlicher Bibliotheken im Hinblick auf die Reichweite ihrer Aktivitäten (lokal, regional, überregional), ihre Zielgruppen sowie fachliche Abdeckung, müssen auch die

8 Neuroth (2012), darin der Beitrag von Dickmann und Rienhoff (2012) 227-56. 
Ziele und Maßnahmen im Bereich Forschungsdatenmanagement angepasst sein. Während eine Universitätsbibliothek alle Fachdisziplinen im Blick haben muss und lokal ausgerichtet ist, muss sich eine fachlich fokussierte Spezialbibliothek an den fachspezifischen Gegebenheiten in ihren Disziplinen orientieren. In der Regel ist hier eine überregionale Zuständigkeit gegeben.

Eine wissenschaftliche Bibliothek kann in allen Schritten und Aufgaben des Lebenszyklus von Forschungsdaten beratend tätig sein. In ausgewählten Schritten kann sie den Datenmanagementprozess durch weitere Services auch direkt unterstützen, wie zum Beispiel durch ein Repositorium. Die Leistungen von ZB MED im Bereich des Forschungsdatenmanagements sind organisatorisch im Programmbereich „Open Access - digitale Langzeitarchivierung - Forschungsdatenmanagement“ angesiedelt. Die Open-Access-Aktivitäten ( $\mathrm{zu}$ denen die Forschungsdaten im weiteren Sinne auch gefasst werden, da es hier um eine breite Zugänglichmachung geht) werden im Publikationsportal PUBLISSO zusammengefasst.

\subsection{1 Überblick}

Das Leistungsspektrum lässt sich grob in die Bereiche Beratungsleistungen, Publikationsdienstleistungen sowie Leistungen zur Verbesserung der Auffindbarkeit und Unterstützung beim Management von Forschungsdaten unterteilen. Begleitet werden die Bereiche durch intensive Vernetzungsarbeit, d. h. Beteiligung von ZB MED in Gremien, z. B. in themenbezogenen Arbeitsgruppen, bzw. den Aufbau von Netzwerken in den wissenschaftlichen Fachcommunities, beispielsweise durch Vorträge auf Fachtagungen. Insgesamt dient der Bereich „Vernetzen“ dazu, eine Verbindung zwischen Theorie und Praxis zu schaffen und die Beratungs- und Publikationsangebote an aktuelle Entwicklungen und die Bedürfnisse der Zielgruppen anzupassen. Da es sich nicht um Dienstleistungen im eigentlichen Sinne, sondern um begleitende Aktivitäten handelt, werden diese nachfolgend nicht weiter aufgeführt. Die Weiterentwicklung von Services erfolgt, indem gezielt Drittmittelprojekte eingeworben werden. Deren Ergebnisse werden in konkrete und nachhaltige Dienstleistungen überführt.

\subsubsection{Beratungsdienstleistungen}

Die Beratung erfolgt über unterschiedliche Formate, die sich in „aktive“ und „passive“ Formate unterteilen lassen. Unter „passive“ Formate werde solche gefasst, die sich zum Selbststudium eignen und bei denen im ersten Schritt keine Interaktion vorgesehen ist. Hierzu zählen Informationstexte in Form von Frequently Asked Questions (FAQs) sowie eigene Tutorials $\mathrm{zu}$ verschiedenen Services im Forschungsdatenmanagement. ${ }^{9}$ Um die Orientierung im Vorfeld einer Forschungsdatenpublikation zu erleichtern, bietet ZB MED zudem eine Übersicht von für die Lebenswissenschaften relevanten Forschungsdatenrepositorien an. ${ }^{10}$ Hierbei handelt es sich um eine Auswahl von Repositorien, die in der Plattform re3data ${ }^{11}$ nachgewiesen werden. Die Webseiten der Repositorien werden geprüft und in Form einer Tabelle nachgewiesen, die nach bestimmten Kriterien wie „fachlicher Schwerpunkt“ oder „Sitz des Betreibers" gefiltert werden kann. Eigene Recherchen sowie ständige Überprüfungen der gelisteten Repositorien ergänzen und aktualisieren die Auflistung.

Bei den derzeit von ZB MED angebotenen „aktiven“ Formaten ist zunächst die persönliche Beratung zu nennen, die telefonisch, per E-Mail oder über persönliche Gespräche erfolgt. Beantwortet werden Fragen zu Veröffentlichung von Forschungsdaten und damit zusammenhängende Themen, Erstellung von Datenmanagementplänen, rechtliche Aspekte etc. Darüber hinaus werden Workshops und Vorträge angeboten, die zielgruppenspezifisch und an sonstige Bedarfe (z. B. zeitlich, Detaillierungsgrad) angepasst werden können. Zielgruppen sind hierbei Wissenschaftler sowie Multiplikatoren in wissenschaftlichen Bibliotheken, an Graduiertenschulen und an lebenswissenschaftlichen Fachbereichen von Hochschulen. Eine Mischform zwischen passiven und aktiven Formaten stellen Webinare dar, die sich bei ZB MED derzeit im Aufbau befinden. Zwar sind die Webinare in erster Linie zum Zuhören und Schauen gedacht, werden aber auch immer interaktive Elemente enthalten und beispielsweise den Teilnehmenden die Möglichkeit eröffnen, Fragen zu stellen.

\subsubsection{Forschungsdaten auffindbar und zitierbar machen: Persistente Identifikatoren}

Durch die Vergabe eines Persistenten Identifikators (PID), z.B. dem Digital Object Identifier (DOI), wird die Auffindbarkeit und Zitierfähigkeit von Publikationen, insbesondere auch Forschungsdaten, sichergestellt. ZB MED ist Mitglied im DataCite-Konsortium und agiert als DOI-Vergabestelle für akademische Onlineangebote aus den Le-

9 www.publisso.de/open-access-beraten/video-tutorials. 10 www.publisso.de/open-access-publizieren/forschungsdaten/for schungsdatenrepositorien.

11 https://www.re3data.org. 
benswissenschaften. DOIs werden schwerpunktmäßig für Forschungsdaten, aber auch für Volltexte vergeben.

Ergänzt werden soll der PID-Service künftig durch die Möglichkeit einen PID zu erhalten, der nicht direkt zu einer Publikation der Forschungsdaten führt, wie in der Regel ein DOI. Ein Beispiel hierfür ist der epicPID. Dieser eignet sich für Arbeitsdaten oder sensitive Daten, die aus Datenschutzgründen nicht veröffentlicht werden können.

\subsubsection{Forschungsdaten publizieren: Publikationsinfrastruktur}

Die Publikationsangebote für Forschungsdaten im Rahmen von PUBLISSO - dem Open-Access-Publikationsportal von ZB MED ${ }^{12}$ bauen auf dem strategischen Ziel auf, die Forschenden zur Nutzung bereits vorhandener Infrastrukturen zur Datenpublikation in den Lebenswissenschaften $\mathrm{zu}$ animieren und an den Stellen eigene Angebote aufzubauen, wo Lücken identifiziert werden. Dies bezieht sich beispielsweise auf den sog. Long Tail der Forschungsdaten, also Daten, die ein geringes Datenvolumen aufweisen, in verschiedenen Datenformaten vorliegen und somit nur schwer standardisierbar sind; aber auch auf lebenswissenschaftliche Teildisziplinen, in denen Möglichkeiten zur Datenarchivierung und -publikation weitgehend fehlen.

Konkrete Angebote im Rahmen der Publikation von Forschungsdaten macht ZB MED in den im Folgenden dargestellten Bereich.

\subsubsection{Publikation von Forschungsdaten im Fachrepositorium Lebenswissenschaften}

Neben der Publikation digitaler Grauer Literatur, Zweitveröffentlichungen und Dissertationen besteht im Fachrepositorium die Möglichkeit, Forschungsdaten „stand-alone“ zu veröffentlichen. Nach Absprache mit dem Zeitschriftenbetreiber ist es auch möglich, Forschungsdaten abzulegen, auf die im Rahmen eines Journalartikels Bezug genommen wird, sofern die Zeitschrift keine eigene Infrastruktur dafür bereithält. Die Datensätze werden so für das Peer Review zugänglich gemacht. Dabei werden folgende Ziele verfolgt: dauerhafte Archivierung von Forschungsdaten aus den Lebenswissenschaften, Sicherstellung der Nachnutzbarkeit der Forschungsdaten, Publikation singulärer Forschungsdaten („raw research data“) sowie von Forschungsdaten, die mit einem Volltext verknüpft sind

12 www.publisso.de. (,enhanced publication“) und die Lizenzierung der Daten im Sinne von Open Data.

Diese Publikationsmöglichkeit für Forschungsdaten wird auch im Rahmen von ZB MED Publikationsinfrastruktur für den sog. goldenen Weg im Open Access genutzt. Das bedeutet, dass Forschungsdaten, die zu einem Zeitschriftenartikel oder einem Buchartikel veröffentlicht werden sollen, ebenfalls im Fachrepositorium Lebenswissenschaften publiziert werden.

\subsubsection{Forschungsdaten finden: Zugriff auf Forschungsdaten über das Suchportal LIVIVO}

Das ZB MED Suchportal für Lebenswissenschaften LIVIVO weist derzeit bereits eine Vielzahl von Forschungsdaten von DataCite Datenzentren (z. B. Beijing Genomics Institute, Natural Environment Research Council) aus den lebenswissenschaftlichen Bereich nach. Diese sind durch den Filter „Documenttype=research data“ direkt suchbar. Geplant ist eine weitere Einbindung von Daten aus lebenswissenschaftlichen Datenrepositorien, vorzugsweise von Datenzentren, die über DataCite DOIs für ihre Forschungsdaten vergeben. Durch die DataCite-Mitgliedschaft von ZB MED ist eine direkte Einbindung über den DOI möglich und die Datenzentren können nach passender fachlicher Ausrichtung und der Bereitstellung geeigneter Forschungsdaten ausgewählt werden. Die über PUBLISSO gold und das Fachrepositorium Lebenswissenschaften publizierten Forschungsdaten werden ebenfalls in LIVIVO nachgewiesen, um die Auffindbarkeit zusätzlich zu steigern.

\subsubsection{Forschungsdaten managen: Tools zur Erstellung eines Datenmanagementplans}

Inzwischen gibt es verschiedene Softwaretools, die die digitale Erstellung eines Datenmanagementplans unterstützen. Vom britischen Digital Curation Center (DCC) wird zum Beispiel die Anwendung DMPonline betrieben. Hier können kollaborativ DMP erstellt und bearbeitet werden, jedoch ist die Anwendung auf die Situation im Vereinigten Königreich mit seinen Förderorganisationen ausgerichtet. Neben den britischen Förderprogrammen lässt sich DMPonline auch für DMP für Horizon 2020 nutzen. Für den deutschsprachigen Raum hat die Humboldt-UniversitätBerlin eine Handreichung zur Benutzung von DMPonline für Horizon 2020 erarbeitet. ${ }^{13}$

13 Simukovic (2014). 
ZB MED konzentriert sich auf das seit 2015 von der DFG geförderte Projekt „Research Data Management Organiser" ${ }^{14}$ das ein generisches Tool zur Erstellung von Datenmanagementplänen entwickelt, das die strukturierte Planung, Umsetzung und Verwaltung des Forschungsdatenmanagements unterstützt und zusätzlich die textuelle Ausgabe eines Forschungsdatenmanagementplans ermöglicht. Es ermöglicht die projektbezogene Erstellung und dynamische Fortschreibung eines Datenmanagementplans. ZB MED hat für das webbasierte Tool eine eigene Instanz RDMO4Life ${ }^{15}$ aufgesetzt, um eine auf die Lebenswissenschaften angepasste Version weiterzuentwickeln.

\subsubsection{Begleitung von Forschungsprojekten durch systematisches Forschungsdatenmanagement: EmiMin-Verbundvorhaben: Emissionsminderung in der Nutztierhaltung}

Neben den Forschungsprojekten, die durch den Bereich Forschung bei ZB MED mit Schwerpunkt in der Informatik durchgeführt werden, beteiligt sich die Abteilung Forschungsdatenmanagement an Projekten aus den Lebenswissenschaften, um ein systematisches Forschungsdatenmanagement zu fördern.

Ein erstes Projekt stellt hier EmiMin dar, in dem ZB MED die Erstellung eines Datenmanagementplans mittels RDMO4Life sowie die Datenpublikation im Fachrepositorium Lebenswissenschaften im engen Austausch mit den Forschenden übernimmt.

\section{Ausblick}

Die Services und Publikationsinfrastrukturen im Leistungsportfolio einer wissenschaftlichen Bibliothek müssen auf die Bedarfe der Fachcommunities ausgerichtet sein. Bei ZB MED hat daher der Aspekt der Vernetzung eine hohe Bedeutung. Weiterhin spielt die Vermittlung von Informationskompetenz - hier die Kompetenz des Datenmanagements - eine entscheidende Rolle.

Durch die Etablierung des Bereichs Forschung bei ZB MED im Rahmen von drei Professuren mit Schwerpunkten in der Informatik und Informationskompetenz werden darüber hinaus künftig Forschungsergebnisse, die das Forschungsdatenmanagement betreffen, in die Services integriert werden.
Beispiel hierfür ist die Verknüpfung heterogener $\mathrm{Da}$ ten unterschiedlicher Teildisziplinen der Lebenswissenschaften durch die Verwendung einheitlicher Metadatenstandards und Terminologien bzw. deren Mapping. Ein weiterer Forschungsansatz beschäftigt sich mit der Erarbeitung von Qualitätskriterien für die Publikation von Forschungsdaten.

Das Leistungsspektrum von ZB MED in Bezug auf das Forschungsdatenmanagement bewegt sich folglich zwischen den Bedarfen der lebenswissenschaftlich Forschenden, der Beratung und Schulung der Forschenden und der Integration relevanter Forschungsergebnisse.

\section{Literaturverzeichnis}

Deutscher Bibliotheksverband (2018): Wissenschaftliche Bibliotheken 2025 (Papier für die Sektion 4 des dbv, Oktober 2017). Verfügbar unter https://www.bibliotheksverband.de/fileadmin/ user_upload/Sektionen/sektion4/Publikationen/WB2025_Endf assung_endg.pdf, veröffentlicht am 2018.

European Commission and Union (2017): EOSC Declaration. Verfügbar unter https://ec.europa.eu/research/openscience/pdf/eosc_de claration.pdf.

Hodson, Simon; Jones, Sarah; Collins, Sandra; Genova, Françoise; Harrower, Natalie; Mietchen, Daniel; Petrauskaité, Ruta; Wittenburg, Peter (2018): Fair Data Action Plan: Interim Recommendations And Actions From The European Commission Expert Group On Fair Data.

Neuroth, Heike (Hrsg.) (2012): Langzeitarchivierung von Forschungsdaten: Eine Bestandsaufnahme. Boizenburg: vwh Hülsbusch.

Rat für Informationsinfrastrukturen (2016a): Leistung aus Vielfalt. Empfehlungen zu Strukturen, Prozessen und Finanzierung des Forschungsdatenmanagements in Deutschland. Verfügbar unter https://d-nb.info/1104292440/34.

Rat für Informationsinfrastrukturen (2016b): Schritt für Schritt - oder: Was bringt wer mit? Ein Diskussionsimpuls zu Zielstellung und Voraussetzungen für den Einstieg in die Nationale Forschungsdateninfrastruktur (NFDI). Verfügbar unter https://d-nb.info/113 $1083113 / 34$.

Rat für Informationsinfrastrukturen (2018): Zusammenarbeit als Chance. Zweiter Diskussionsimpuls zur Ausgestaltung einer Nationalen Forschungsdateninfrastruktur (NFDI) für die Wissenschaft in Deutschland. Verfügbar unter https://d-nb.info/11544 $6217 X / 34$.

Simukovic, Elena (2014): Anleitung zur Erstellung eines Datenmanagementplans (DMP) in Horizon 2020. Verfügbar unter https:// www.cms.hu-berlin.de/de/dl/dataman/dmp-anleitung.

14 https://rdmorganiser.github.io.

15 rdmo.publisso.de. 


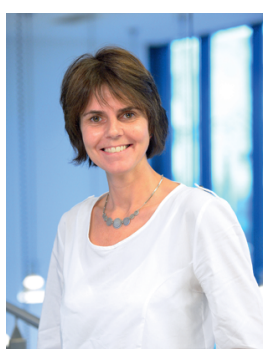

Birte Lindstädt

PUBLISSO - Forschungsdatenmanagement

Open Access - Digitale

Langzeitarchivierung -

ForschungsdatenmanagementZB MED -

Informationszentrum

Lebenswissenschaften

Gleueler Straße 60

D-50931 Köln

lindstaedt@zbmed.de

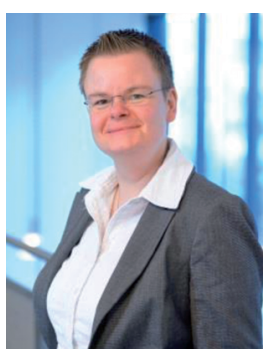

Jasmin Schmitz

PUBLISSO - Publikationsberatung

Open Access - Digitale Langzeitarchi-

vierung - Forschungsdatenmanagement

ZB MED - Informationszentrum

Lebenswissenschaften

Gleueler Straße 60

D-50931 Köln

schmitz@zbmed.de 